Supporting information

\title{
Resistome of Staphylococcus aureus in Response to Human Cathelicidin LL-37 and Its Engineered Antimicrobial Peptides
}

Radha Golla ${ }^{1}$, Biswajit Mishra ${ }^{1}$, Xiangli Dang ${ }^{1, \#}$, Jayaram Lakshmaiah Narayana ${ }^{1}$, Amy Li $^{2}$, Libin $\mathrm{Xu}^{2}$, and Guangshun Wang ${ }^{1, *}$

${ }^{1}$ Department of Pathology and Microbiology, College of Medicine, University of Nebraska Medical Center, 985900 Nebraska Medical Center, Omaha, NE 68198-5900, USA

${ }^{2}$ Department of Medicinal Chemistry, University of Washington, Seattle, WA 98195

\section{Contents}

Supporting Tables S1-S2

Supporting Figures S1-S2 
Table S1. Minimal inhibitory concentrations $(\mu M)$ of LL-37 peptides and antibiotics used in this study ${ }^{1}$

\begin{tabular}{|c|c|c|}
\hline Antimicrobial compound & S. aureus JE2 & S. aureus USA300 \\
\hline LL-37 & 3.1 & 3.1 \\
\hline GF-17 & 3.1 & 3.1 \\
\hline 17BIPHE2 & 3.1 & 3.1 \\
\hline DFTamP1 & $1.5-3.1$ & 3.1 \\
\hline Daptomycin & 6.2 & 6.2 \\
\hline Linezolid & 3.1 & 3.1 \\
\hline Tigecycline & 0.37 & 0.37 \\
\hline Tobramycin & $0.75-1.5$ & 0.37 \\
\hline Vancomycin & 0.37 & 1.5 \\
\hline
\end{tabular}

${ }^{1}$ All the measurements were made in 50\% TSB except for human LL-37, which was conducted in $12.5 \%$ TSB. This is because LL-37 is not active in $100 \%$ TSB and the MIC values also depend on the percent of TSB used ${ }^{1}$. The MIC of daptomycin depends on calcium and insufficient $\mathrm{Ca}^{2+}$ could lead to higher MIC values. However, our focus here is on the MIC differences between the wild type JE2 and the mutants.

Reference 1 .

Mishra, B., Golla, R.M., Lau, K., Lushnikova, T., Wang, G. Anti-staphylococcal biofilm effects of human cathelicidin peptides. ACS Med Chem Lett. 2016, 7(1), 117-121. 
Table S2. Nucleotide Sequences of Primers Used in This Study ${ }^{1}$

\begin{tabular}{|c|c|c|c|c|c|}
\hline Gene id & $\begin{array}{l}\text { Primer } \\
\text { name }\end{array}$ & Primer sequences (5'-3') & $\begin{array}{l}\text { Gene } \\
\text { size } \\
\text { (bp) }\end{array}$ & $\begin{array}{l}\text { Tn } \\
\text { size } \\
\text { (bp) }\end{array}$ & $\begin{array}{l}\text { Calculatec } \\
\text { size (bp) }\end{array}$ \\
\hline \multirow[b]{2}{*}{$\begin{array}{l}\text { SAUSA300 } \\
-1898\end{array}$} & $1898-\mathrm{F}$ & CGTGGAAGATGTAAACCAAC & \multirow[b]{2}{*}{174} & \multirow[b]{2}{*}{133} & \multirow[b]{2}{*}{307} \\
\hline & Buster & $\begin{array}{l}\text { GCTTTTTCTAAATGTTTTTTAA } \\
\text { GTAAATCAAGTAC }\end{array}$ & & & \\
\hline \multirow{2}{*}{$\begin{array}{l}\text { SAUSA300 } \\
1503\end{array}$} & $1503-\mathrm{F}$ & ACTATTAAGCAACAGCGACA & \multirow{2}{*}{224} & \multirow{2}{*}{464} & \multirow{2}{*}{688} \\
\hline & Upstream & CTCGATTCTATTAACAAGGG & & & \\
\hline \multirow[b]{2}{*}{$\begin{array}{l}\text { SAUSA300 } \\
\quad 1867\end{array}$} & $1867-\mathrm{F}$ & GCATAGAAAGGCGGCGAAAC & \multirow[b]{2}{*}{148} & \multirow[b]{2}{*}{133} & \multirow[b]{2}{*}{281} \\
\hline & Buster & $\begin{array}{l}\text { GCTTTTTCTAAATGTTTTTTAA } \\
\text { GTAAATCAAGTAC }\end{array}$ & & & \\
\hline \multirow{2}{*}{$\begin{array}{l}\text { SAUSA300 } \\
0829\end{array}$} & 0829-F & TCGGTCGTTATTTCCCAACA & \multirow{2}{*}{329} & \multirow{2}{*}{464} & \multirow{2}{*}{793} \\
\hline & Upstream & CTCGATTCTATTAACAAGGG & & & \\
\hline \multirow{2}{*}{$\begin{array}{l}\text { SAUSA300 } \\
1036\end{array}$} & $1036-\mathrm{F}$ & TGAAGATGAAAGTAGCCCGA & \multirow{2}{*}{514} & \multirow{2}{*}{464} & \multirow{2}{*}{978} \\
\hline & Upstream & CTCGATTCTATTAACAAGGG & & & \\
\hline \multirow{2}{*}{$\begin{array}{l}\text { SAUSA300 } \\
1962 \\
\end{array}$} & $1962-\mathrm{F}$ & ACTCAAGACGAATTAAGGGG & \multirow{2}{*}{322} & \multirow{2}{*}{464} & \multirow{2}{*}{786} \\
\hline & Upstream & CTCGATTCTATTAACAAGGG & & & \\
\hline \multirow{2}{*}{$\begin{array}{l}\text { SAUSA300 } \\
0647\end{array}$} & $0647-\mathrm{F}$ & TGTCTATTGAAGAAGGCGAG & \multirow[b]{2}{*}{162} & \multirow[b]{2}{*}{133} & \multirow[b]{2}{*}{295} \\
\hline & Buster & $\begin{array}{l}\text { GCTTTTTCTAAATGTTTTTTAA } \\
\text { GTAAATCAAGTAC }\end{array}$ & & & \\
\hline \multirow{2}{*}{$\begin{array}{l}\text { SAUSA300 } \\
1255\end{array}$} & $1255-\mathrm{F}$ & GCTTTATTCCTGGTGGTTTC & & & \\
\hline & Upstream & CTCGATTCTATTAACAAGGG & 434 & 404 & 898 \\
\hline & $0186-\mathrm{F}$ & CAGCACCCGTAAGTAAAACA & & & \\
\hline-0186 & Buster & $\begin{array}{l}\text { GCTTTTTCTAAATGTTTTTTAA } \\
\text { GTAAATCAAGTAC }\end{array}$ & 380 & 133 & 513 \\
\hline SAUSA300 & $0646-\mathrm{F}$ & CGCCATTTCAACGTCATACA & 518 & 464 & 982 \\
\hline & Upstream & CTCGATTCTATTAACAAGGG & 310 & 404 & 902 \\
\hline SAUSA300 & $1465-\mathrm{F}$ & TACTACTCAAGGACTGCAAC & 266 & 464 & 730 \\
\hline 1465 & Upstream & CTCGATTCTATTAACAAGGG & 206 & & \\
\hline & $1865-\mathrm{F}$ & TCAGGTACACGTATCGAGGT & & & \\
\hline $\begin{array}{l}\text { SAUSA300 } \\
\text { - } 1865\end{array}$ & Buster & $\begin{array}{l}\text { GCTTTTTCTAAATGTTTTTTAA } \\
\text { GTAAATCAAGTAC }\end{array}$ & 137 & 133 & 270 \\
\hline SAUSA300 & $1097-\mathrm{F}$ & GGTTTAGATGGCGTTGTTTG & 62 & 464 & \\
\hline 1097 & Upstream & CTCGATTCTATTAACAAGGG & 02 & 404 & \\
\hline & $1228-\mathrm{F}$ & TAGTGAACCAGCGAGTAATG & & & \\
\hline-1228 & Buster & $\begin{array}{l}\text { GCTTTTTCTAAATGTTTTTTAA } \\
\text { GTAAATCAAGTAC }\end{array}$ & 405 & 133 & 538 \\
\hline & $1967-\mathrm{F}$ & GCTCCTTTCTGCTATACTCC & & & \\
\hline $\begin{array}{l}\text { SAUSA300 } \\
-1967\end{array}$ & Buster & $\begin{array}{l}\text { GCTTTTTCTAAATGTTTTTTAA } \\
\text { GTAAATCAAGTAC }\end{array}$ & 191 & 133 & 324 \\
\hline SAUSA300 & $0645-\mathrm{F}$ & AGATTTCGGCAAAGTAATGG & 12 & & \\
\hline 0645 & Upstream & CTCGATTCTATTAACAAGGG & 125 & 404 & 389 \\
\hline SAUSA300 & 0394-F & TACAGGTCATTTAGGCACAC & 53 & 464 & 517 \\
\hline
\end{tabular}




\begin{tabular}{|c|c|c|c|c|c|}
\hline 0394 & Upstream & CTCGATTCTATTAACAAGGG & & & \\
\hline \multirow{2}{*}{$\begin{array}{l}\text { SAUSA300 } \\
1308\end{array}$} & $1308-\mathrm{F}$ & GGACACAGAGTATGATGGA & \multirow{2}{*}{405} & \multirow{2}{*}{464} & \multirow{2}{*}{869} \\
\hline & Upstream & CTCGATTCTATTAACAAGGG & & & \\
\hline \multirow{2}{*}{$\begin{array}{l}\text { SAUSA300 } \\
-1469\end{array}$} & $1469-\mathrm{F}$ & GTCGTAAATCAGTGCATGTGT & \multirow[b]{2}{*}{85} & \multirow[b]{2}{*}{133} & \multirow[b]{2}{*}{218} \\
\hline & Buster & $\begin{array}{l}\text { GCTTTTTCTAAATGTTTTTTAA } \\
\text { GTAAATCAAGTAC }\end{array}$ & & & \\
\hline \multirow{2}{*}{$\begin{array}{l}\text { SAUSA300 } \\
\text { _0988 }\end{array}$} & 0988-F & TAAGTTGAGACGATACCCCA & \multirow[b]{2}{*}{153} & \multirow[b]{2}{*}{133} & \multirow[b]{2}{*}{286} \\
\hline & Buster & $\begin{array}{l}\text { GCTTTTTCTAAATGTTTTTTAA } \\
\text { GTAAATCAAGTAC }\end{array}$ & & & \\
\hline \multirow{2}{*}{$\begin{array}{l}\text { SAUSA300 } \\
1089 \\
\end{array}$} & $1089-\mathrm{F}$ & CACATCGAAATAATGGTGCT & \multirow{2}{*}{145} & \multirow{2}{*}{464} & \multirow{2}{*}{609} \\
\hline & Upstream & CTCGATTCTATTAACAAGGG & & & \\
\hline \multirow{2}{*}{$\begin{array}{l}\text { SAUSA300 } \\
-1515\end{array}$} & $1515-\mathrm{F}$ & TGGACATCCTGTACGTTTTG & \multirow[b]{2}{*}{214} & \multirow[b]{2}{*}{133} & \multirow[b]{2}{*}{347} \\
\hline & Buster & $\begin{array}{l}\text { GCTTTTTCTAAATGTTTTTTAA } \\
\text { GTAAATCAAGTAC }\end{array}$ & & & \\
\hline \multirow{2}{*}{$\begin{array}{l}\text { SAUSA300 } \\
1336\end{array}$} & $1336-\mathrm{F}$ & GTAACTTCCCAGTTCAAGGT & \multirow{2}{*}{498} & \multirow{2}{*}{464} & \multirow{2}{*}{962} \\
\hline & Upstream & CTCGATTCTATTAACAAGGG & & & \\
\hline
\end{tabular}

${ }^{1}$ These primers were designed to validate the insertion of the transposon (Tn). 


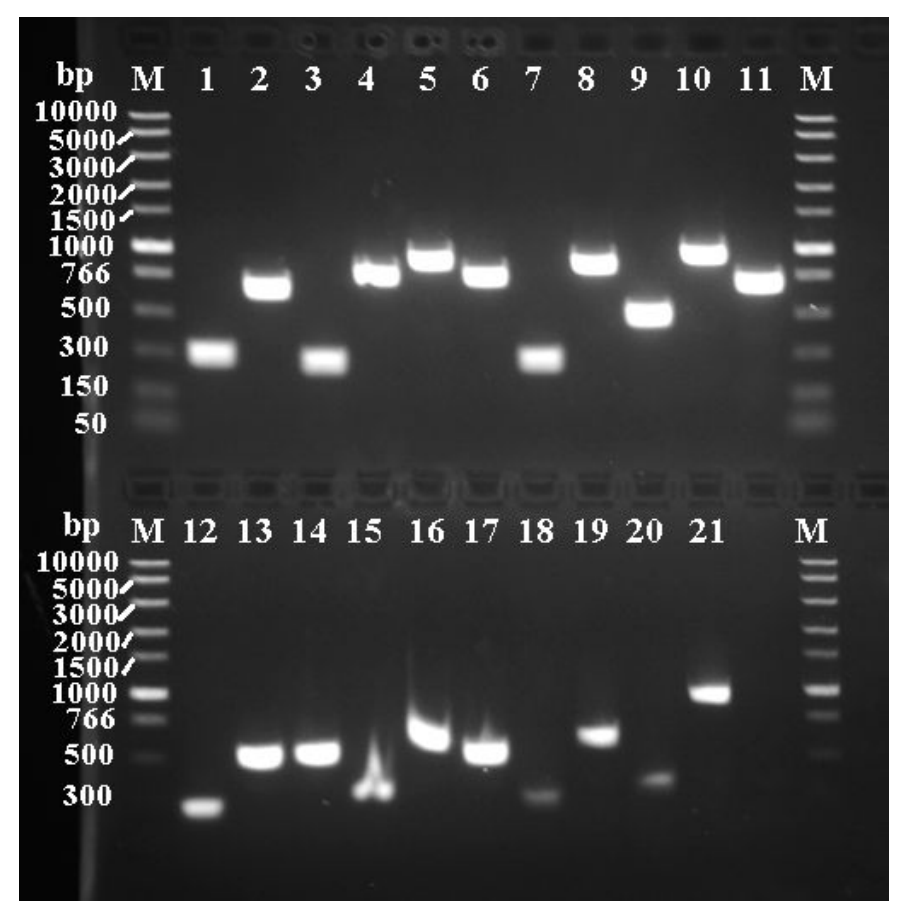

Figure S1. Agarose gel (1\%) electrophoresis of PCR products of the susceptible mutants identified from the library of $S$. aureus JE2. M: Fast DNA marker (NEB, USA). 1:

USA300_1898; 2: USA300_1503; 3: USA300_1867; 4: USA300_0829; 5: USA300_1036; 6: USA300_1962; 7: USA300_0647; 8: USA300_1255; 9: USA300_0186; 10:USA300_0646; 11: USA300_1465; 12:USA300_1865; 13: USA300_1097; 14: USA300_1228; 15: USA300_1967; 16:0645; 17: USA300_0394; 18: USA300_0988; 19: USA300_1089; 20: USA300_1515; 21: USA300_1336. 

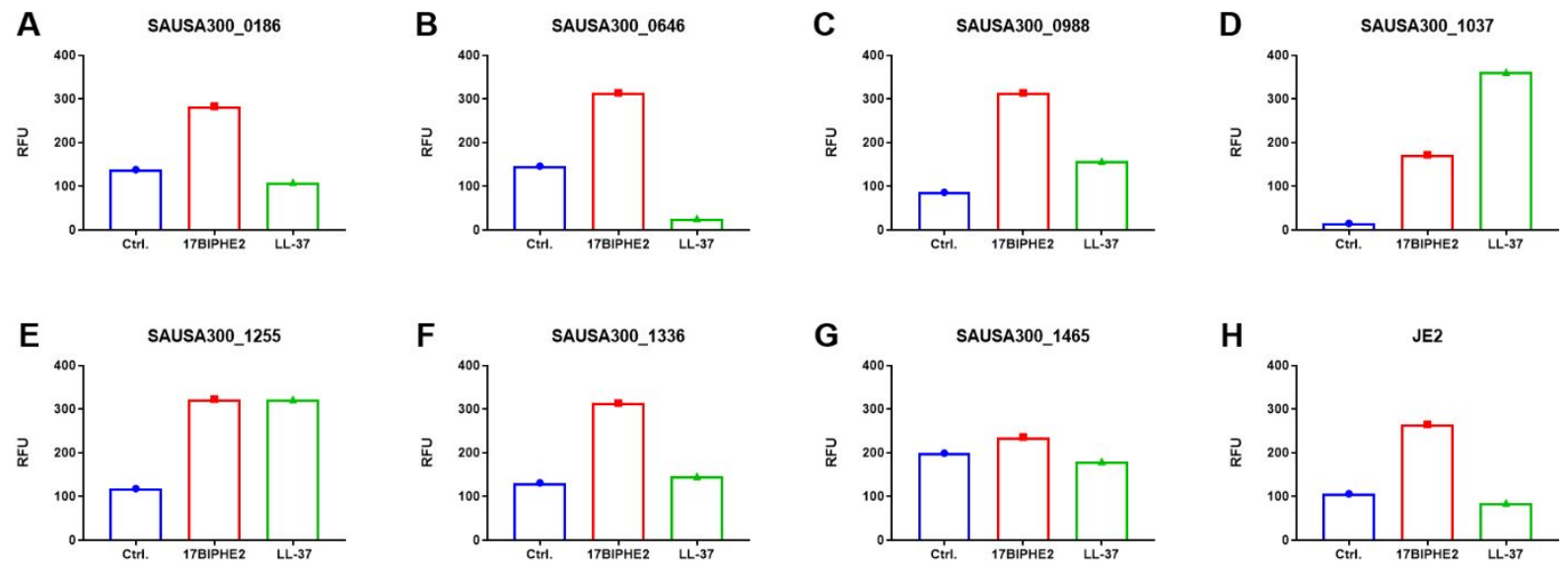

Fig. S2 Panel 1. Membrane permeation amplitude plots at 15 min based on Fig. 3 panel 1. These plots indicate that the membranes of S. aureus became more permeable to 17BIPHE2 in most of the cases (e.g. A, B, C, F, and G), similar to the wild type $S$. aureus JE2 (H). However, LL-37 is more powerful than 17BIPHE2 in D. Finally, we observed a similar amplitude of fluorescence in $\mathbf{E}$, all at $15 \mathrm{~min}$. 

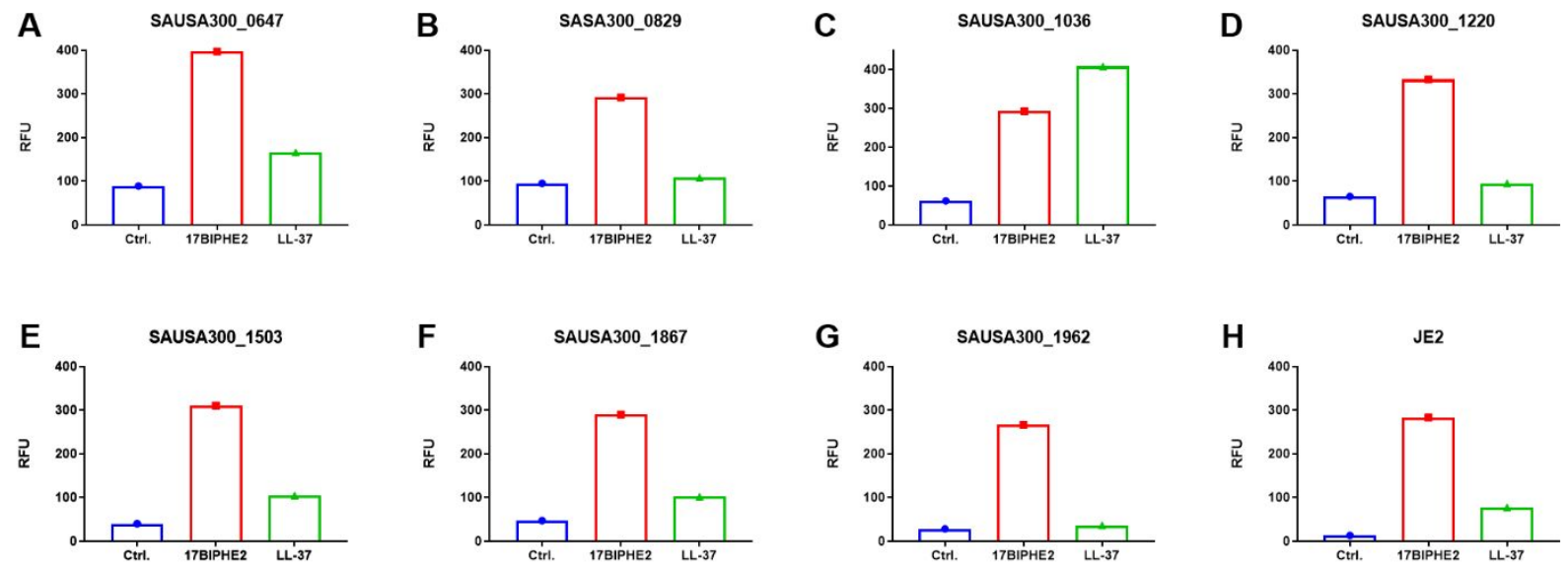

Fig. S2 Panel 2. Membrane permeation amplitude plots at 15 min based on Fig. 3 panel 2. These plots indicate that the membranes of S. aureus became more permeable to 17BIPHE2 in most of the cases (e.g. A, B, D, E, F, and G), similar to the wild type S. aureus JE2 (H). However, LL37 is slightly more powerful than 17BIPHE2 in $\mathbf{C}$, all at $15 \mathrm{~min}$. 

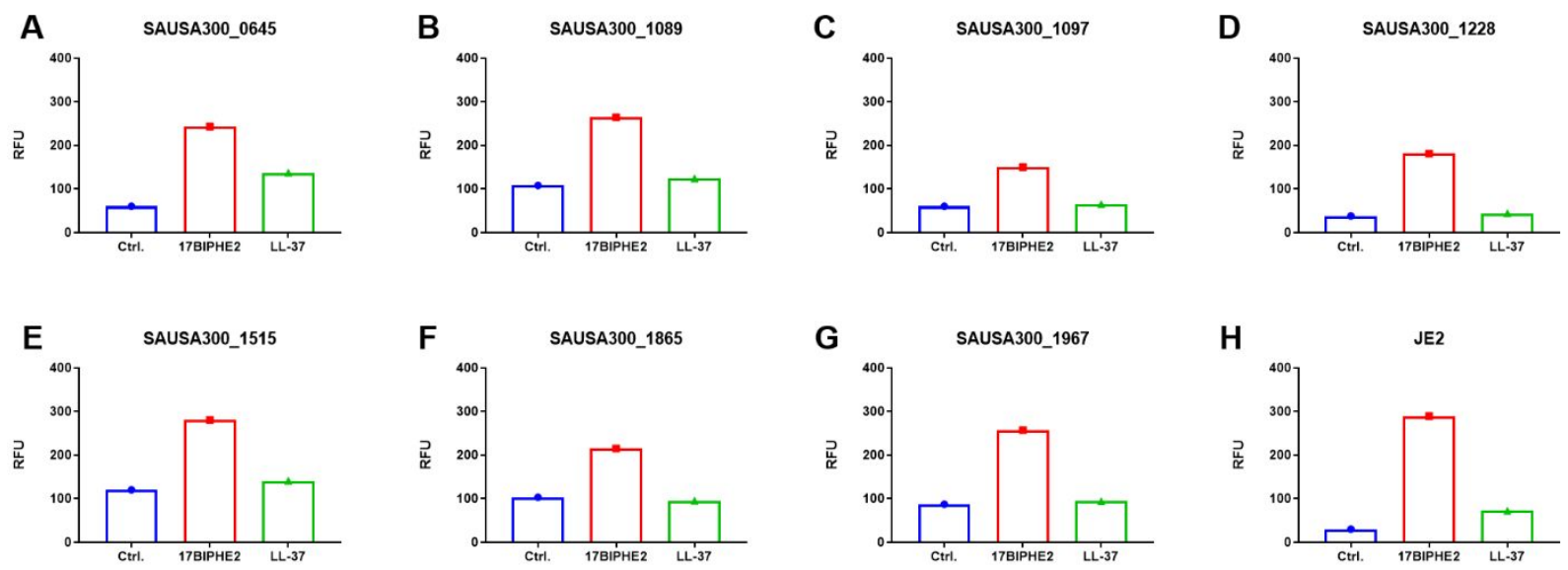

Fig. S2 Panel 3. Membrane permeation amplitude plots at 15 min based on Fig. 3 panel 3. These plots indicate that the membranes of S. aureus became more permeable to 17BIPHE2 in all the cases (A-G), similar to the wild type S. aureus JE2 (H) at $15 \mathrm{~min}$. 